\title{
Anticoagulation and Mortality Rates among Hospitalized Patients with Atrial Fibrillation
}

\author{
Gregory Piazza ${ }^{1}$ Shelley Hurwitz ${ }^{2}$ Lindsay M. Harrigan ${ }^{1}$ Kathryn L. Jenkins ${ }^{1}$ Benjamin Hohlfelder ${ }^{3}$ \\ John Fanikos ${ }^{3}$ Samuel Z. Goldhaber ${ }^{1}$
}

${ }^{1}$ Cardiovascular Division, Department of Medicine, Brigham and Women's Hospital, Harvard Medical School, Boston, Massachusetts, United States

${ }^{2}$ Center for Clinical Investigation, Brigham and Women's Hospital, Boston, Massachusetts, United States

${ }^{3}$ Department of Pharmacy, Brigham and Women's Hospital, Boston, Massachusetts, United States

TH Open 2018;2:e33-e38.
Address for correspondence Gregory Piazza, MD, MS, Cardiovascular Division, Brigham and Women's Hospital, 75 Francis Street, Boston, MA 02115, United States (e-mail: gpiazza@partners.org).

\begin{abstract}
Keywords

- anticoagulation

- atrial fibrillation

- mortality

- adverse events

Atrial fibrillation (AF) is associated with an increased rate of mortality, heart failure, and stroke. We conducted an observational study to assess the relationship between anticoagulation and adverse clinical outcomes in hospitalized patients with AF. We performed a 5,000-consecutive-patient retrospective cohort analysis of anticoagulation prescription and 90-day outcomes in patients with AF hospitalized at Brigham and Women's Hospital from May 2008 to September 2014. All-cause mortality at 90 days was $5.4 \%$. The frequency of death between hospital discharge and day 90 was lower in patients who were anticoagulated at discharge ( 2.8 vs. $7.1 \%, p<0.001)$. Anticoagulation prescription at discharge was associated with a $60 \%$ reduction in death between discharge and day 90 , after adjustment for confounding factors. Major adverse events at day 90, including death, myocardial infarction, stroke, and major bleeding, were more frequent in patients who were not prescribed anticoagulation at discharge (16.5 vs. $10.4 \%, p<0.001)$. In multivariable regression analysis, prescription of anticoagulation at discharge predicted a lower mortality (adjusted odds ratio (OR), 0.4; $95 \%$ confidence interval $(\mathrm{Cl}), 0.3-0.53)$ and lower major adverse event rate (adjusted $\mathrm{OR}, 0.64 ; 95 \% \mathrm{Cl}, 0.54-0.76$ ) by day 90 . In conclusion, all-cause mortality at 90 days was high among inpatients with AF. Patients with AF who were not prescribed anticoagulation at discharge had an increased risk of death at 90 days. Hospitalization represents a special opportunity to optimize cardiovascular risk reduction strategies, including anticoagulation.
\end{abstract}

\section{Introduction}

In 1998, the Framingham Heart Study reported that atrial fibrillation (AF) had a multivariable-adjusted association with an increased risk of death, ${ }^{1}$ and this was subsequently corroborated. ${ }^{2-4}$ A meta-analysis of 1,009,501 patients, of whom 149,746 had AF, found a $60 \%$ increased risk of death in $\mathrm{AF}$, primarily due to increased cardiovascular mortality. ${ }^{5} \mathrm{~A}$ separate meta-analysis of antithrombotic studies showed a $1.6 \%$ absolute risk reduction of all-cause mortality in patients with $\mathrm{AF}$ who received antithrombotic therapy. ${ }^{6}$ While anticoagulation prevents stroke in patients with $\mathrm{AF}$, it may also reduce the risk of other major adverse cardiovascular outcomes. ${ }^{7}$ To follow up these previous studies, we assessed the relationship between anticoagulation prescription and fatal and nonfatal cardiovascular outcomes and major adverse received

July 17, 2017

accepted after revision

December 19, 2017
DOI https://doi.org/

10.1055/s-0038-1626732. ISSN 2512-9465.
๑) 2018 Georg Thieme Verlag KG
Stuttgart · New York

License terms

(1)(1) 
clinical events among 5,000 hospitalized patients with AF at Brigham and Women's Hospital (BWH).

\section{Materials and Methods}

\section{Study Oversight}

The study was conducted according to the ethical principles stated in the Declaration of Helsinki. Institutional Review Board approval was obtained. The requirement of informed consent was waived because the study was a quality improvement initiative and a medical record review.

\section{Study Design}

The study was a retrospective cohort analysis using data abstracted through our electronic health record (EHR) at BWH.

\section{Patient Population}

BWH is a 777-bed acute tertiary care facility. Consecutive patients, aged 18 years or older, who were hospitalized at BWH between May 4, 2008, and September 30, 2014, with an admitting diagnosis of $\mathrm{AF}$, were included. For patients with multiple admissions due to AF, only the first admission was included. Patients with valvular heart disease graded "severe" or those with mechanical prosthetic heart valves were excluded.

\section{Data Query and Collection}

Study data were collected by trained research staff (L.M.H., K. L.J., and B.H.) and managed using the Research Electronic Data Capture (REDCap) electronic data capture tool hosted at $\mathrm{BWH} .{ }^{8}$ REDCap is a secure, web-based application designed to support data capture for research studies.

Patient demographics and baseline clinical characteristics were recorded, including age, gender, race, ethnicity, and comorbid conditions. Clinical characteristics of AF, including symptoms and rhythm management, individual risk factors for stroke and bleeding, and $\mathrm{CHA}_{2} \mathrm{DS}_{2}-\mathrm{VASc}^{9}$ and HASBLED $^{10}$ scores, were obtained from the EHR. Anticoagulation prescription was defined as any order for therapeutic-dose antithrombotic therapy, including non-vitamin K oral anticoagulants, vitamin $\mathrm{K}$ antagonists (warfarin), intravenous heparin, low-molecular-weight heparins (LMWH), and fondaparinux. Labile INRs, as a component of the HAS-BLED score, was defined as any clinical mention in the EHR of difficulty maintaining the INR within the target range.

Ninety-day clinical outcomes of acute coronary syndrome, stroke, bleeding events, and all-cause mortality were obtained for all patients by utilizing our EHR, which captures any patient encounter across 6 affiliated hospitals, 17 ambulatory clinics, and numerous private practices. Patient encounters were recorded in the form of discharge summaries, office notes, diagnostic testing reports, medical treatment summaries, and procedure reports, regardless of the facility or office location. Bleeding events were classified according to the International Society of Thrombosis and Haemostasis (ISTH) criteria for major and nonmajor bleeding. ${ }^{11}$ The Social Security Death Index (SSDI) was used to identify patients who died during the 90-day follow-up period. Mortality outcomes were confirmed using the Center for Disease Control (CDC) National Death Index (NDI). Causes of death were categorized as cardiovascular or noncardiovascular. The frequency of major adverse events at 90 days, including acute coronary syndrome, stroke, bleed, or death, was recorded. Ninety-day follow-up was completed for $100 \%$ of study patients.

\section{Statistical Analysis}

Descriptive statistics, including baseline demographic and clinical characteristics, assessment of stroke and bleeding risk, patterns of stroke prevention in AF, and 90-day clinical outcomes, were stratified as continuous or binary. Continuous variables were assessed for normality of distribution. Normally distributed continuous variables were presented as means with standard deviations. Nonparametric data were presented as median with interquartile ranges. Binary variables were presented as numbers and proportions. Comparative statistics for categorical variables were calculated using the chi-square test or Fisher's exact test. Comparative statistics for continuous variables were calculated using a two-sample $t$-test or Wilcoxon's rank-sum test.

Multivariate regression analyses were conducted to evaluate whether prescription of anticoagulation was associated with death at discharge, death between discharge and day 90, major adverse events at 90 days, and ISTH major bleeding at 90 days, while controlling for several potentially confounding prognostic factors. Variables included in the regression models were selected based on results of univariate analysis and a priori knowledge and were composed of age, gender, $\mathrm{CHA}_{2} \mathrm{DS}_{2}$-VASc score, and HAS-BLED score.

All reported $p$-values were two sided. All statistical analyses were performed using SAS version 9.4 (SAS Institute, Cary, North Carolina, United States).

\section{Results}

\section{Baseline Demographics and Clinical Characteristics}

We identified 5,000 unique patients hospitalized at BWH with an admitting diagnosis of AF. The mean age was 69 years (-Table 1). The study population was overweight, with a mean body mass index of $29 \mathrm{~kg} / \mathrm{m}^{2}$. Common comorbid conditions included coronary artery disease $(22 \%)$ and cardiomyopathy (13.6\%).

AF was paroxysmal in $40.1 \%$, new in $14.1 \%$, persistent in $10.6 \%$, permanent in $1.4 \%$, and unclassified in $33.8 \%$. Rate and rhythm control were prescribed in 83 and 30\%, respectively.

\section{Risk of Stroke and Bleeding}

The median $\mathrm{CHA}_{2} \mathrm{DS}_{2}$-VASc score was 3 points (-Table 2). The most frequently observed components of the $\mathrm{CHA}_{2} \mathrm{DS}_{2}$ VASc score were hypertension (70.4\%), age $\geq 75$ years (35.9\%), and female gender (37.7\%). The median HAS-BLED score was 3 points.

\section{Clinical Outcomes}

All-cause mortality at 90 days in the overall patient cohort was $5.4 \%$. Cardiovascular causes were noted in $39.2 \%$ of inpatient deaths and $12.2 \%$ of those taking place between 
Table 1 Baseline demographic and clinical characteristics

\begin{tabular}{|c|c|}
\hline Characteristic & $N=5,000$ \\
\hline Mean age \pm standard deviation, y & $69.2 \pm 13.1$ \\
\hline Male, $n(\%)$ & $3,114(62.3)$ \\
\hline \multicolumn{2}{|l|}{ Race/ethnicity, n (\%) } \\
\hline White & $4,394(87.9)$ \\
\hline Black & $268(5.4$ \\
\hline Hispanic/Latino & $161(3.2)$ \\
\hline Asian & $59(1.2)$ \\
\hline Other & $14(0.28)$ \\
\hline Cardiomyopathy, $n(\%)$ & $680(13.6)$ \\
\hline Coronary artery disease, $n(\%)$ & $1,113(22.0)$ \\
\hline $\begin{array}{l}\text { Prior myocardial infarction or } \\
\text { unstable angina }\end{array}$ & $744(14.9)$ \\
\hline Prior coronary intervention & $517(10.3)$ \\
\hline $\begin{array}{l}\text { Prior coronary artery bypass graft } \\
\text { surgery }\end{array}$ & $467(9.3)$ \\
\hline Prior venous thromboembolism, $n$ (\%) & $407(8.1)$ \\
\hline History of falls, $n(\%)$ & $662(13.2)$ \\
\hline Current smoker, n (\%) & $318(6.4)$ \\
\hline Former smoker, $n(\%)$ & $2,188(43.8)$ \\
\hline $\begin{array}{l}\text { Prior hospitalization within prior } \\
30 \mathrm{~d}, n(\%)\end{array}$ & $704(14.1)$ \\
\hline $\begin{array}{l}\text { Chronic obstructive lung disease, } \\
n(\%)\end{array}$ & $527(10.5)$ \\
\hline Chronic kidney disease, $n(\%)$ & $683(13.7)$ \\
\hline Hemodialysis, $n$ (\%) & $55(8.1)$ \\
\hline
\end{tabular}

discharge and day 90 . Stroke occurred in $2.8 \%$, and bleeding events occurred in $4.3 \%$ at 90 days. Ischemic strokes (63.4\%) comprised the majority of cerebrovascular events. ISTH major bleeds comprised $42.6 \%$ of the bleeding events. Half of the bleeding events were spontaneous. The gastrointestinal tract was the most common site of bleeding (24.1\%), followed by surgical (18.1\%) and intracranial (6.5\%). The overall rate of major adverse events at 90 days was $12.4 \%$, including stroke, MI, bleeding, and death. Major adverse events at 90 days increased with higher $\mathrm{CHA}_{2} \mathrm{DS}_{2}$-VASc and HAS-BLED scores (-Fig. 1).

\section{Characteristics and Outcomes of Anticoagulated versus Non-anticoagulated Patients}

Hospitalized patients who were prescribed anticoagulation at discharge were slightly younger (mean age: 68.7 vs. 70.1 years, $p=0.01$ ). Those with cardiomyopathy ( $16.4 \%$ versus $9 \%, p<0.001$ ) or a history of heart failure (21.6 vs. $15.2 \%$, $p<0.001$ ) were more likely to be anticoagulated. In contrast, those with a history of prior falls ( 10.2 vs. $18.2 \%, p<0.001)$ or dementia ( 2.3 vs. $5.2 \%, p<0.001$ ) were less likely to be anticoagulated. The frequency of coronary artery disease was similar between anticoagulated and non-anticoagulated patients ( 27.9 vs. $30 \%, p=0.11$ ).
Table 2 Assessment of stroke and bleeding risk

\begin{tabular}{|c|c|}
\hline Characteristic & $N=5,000$ \\
\hline Heart failure, $n(\%)$ & $955(19.1)$ \\
\hline Hypertension, $n(\%)$ & $3,522(70.4)$ \\
\hline Age $\geq 75$ y, $n(\%)$ & $1,796(35.9)$ \\
\hline Age 65-74 y, $n$ (\%) & $1,439(28.8)$ \\
\hline Female, $n(\%)$ & $1,886(37.7)$ \\
\hline Diabetes, $n(\%)$ & $1,045(20.9)$ \\
\hline $\begin{array}{l}\text { Prior stroke, transient ischemic attack, } \\
\text { or systemic embolism, } n(\%)\end{array}$ & $881(17.6)$ \\
\hline Vascular disease, $n(\%)$ & $1,641(32.8)$ \\
\hline Renal dysfunction, $n$ (\%) & $325(6.5)$ \\
\hline Liver disease, $n(\%)$ & $227(4.5)$ \\
\hline $\begin{array}{l}\text { Prior major bleeding or predisposition } \\
\text { to bleeding, } n(\%)\end{array}$ & $1,512(30.2)$ \\
\hline $\begin{array}{l}\text { Labile international normalized ratio, } \\
n(\%)\end{array}$ & $1,313(26.3)$ \\
\hline $\begin{array}{l}\text { Concomitant antiplatelet therapy or } \\
\text { nonsteroid anti-inflammatory drugs, } \\
n(\%)\end{array}$ & $2,581(51.6)$ \\
\hline $\begin{array}{l}\text { Alcohol intake } \geq 8 \text { servings per week, } \\
n(\%)\end{array}$ & $441(8.8)$ \\
\hline $\begin{array}{l}\text { Median } \mathrm{CHA}_{2} \mathrm{DS}_{2} \text {-VASc score } \\
\text { (interquartile range), points }\end{array}$ & $3(2-4)$ \\
\hline $\begin{array}{l}\text { Median HAS-BLED score } \\
\text { (interquartile range), } \\
\text { points }\end{array}$ & $3(2-4)$ \\
\hline
\end{tabular}

Inpatients who were not prescribed anticoagulation at discharge were more likely to be older, have a prior major bleeding event or predisposition to bleeding, or be prescribed nonsteroidal anti-inflammatory drugs (-Table 3 ). Patients who received inpatient anticoagulation were more likely to be prescribed anticoagulation at discharge compared with those who were not (85.7 vs. $10.4 \%, p<0.001)$.

The frequencies of inpatient mortality ( 2.6 vs. $0.03 \%$, $p<0.001)$ and death between hospital discharge and day 90 ( 7.1 vs. $2.8 \%, p<0.001$ ) were higher in patients not prescribed anticoagulation at discharge. Major adverse events at day 90, including death, myocardial infarction, stroke, and major bleeding, were more frequent in patients not prescribed anticoagulation at discharge (16.5 vs. $10.4 \%$, $p<0.0001)$. Acute coronary syndromes at day 90 occurred with similar frequency among patients who were prescribed anticoagulation and those who were not ( 0.9 vs. $1.3 \%$, $p=0.13)$.

In multivariable regression analysis, prescription of anticoagulation at discharge was associated with lower mortality (adjusted odds ratio [OR], 0.4; 95\% confidence interval [CI], 0.3-0.53), lower ISTH major bleeding (adjusted OR, 0.5; 95\% $\mathrm{CI}, 0.26-0.81$ ), and a lower major adverse event rate (adjusted OR, 0.64; 95\% CI, 0.54-0.76) by day 90. In contrast, increasing $\mathrm{CHA}_{2} \mathrm{DS}_{2}$-VASc (adjusted OR, 1.13; 95\% CI, 1.011.26) and HAS-BLED scores (adjusted OR, 1.16; 95\% CI, 1.01- 


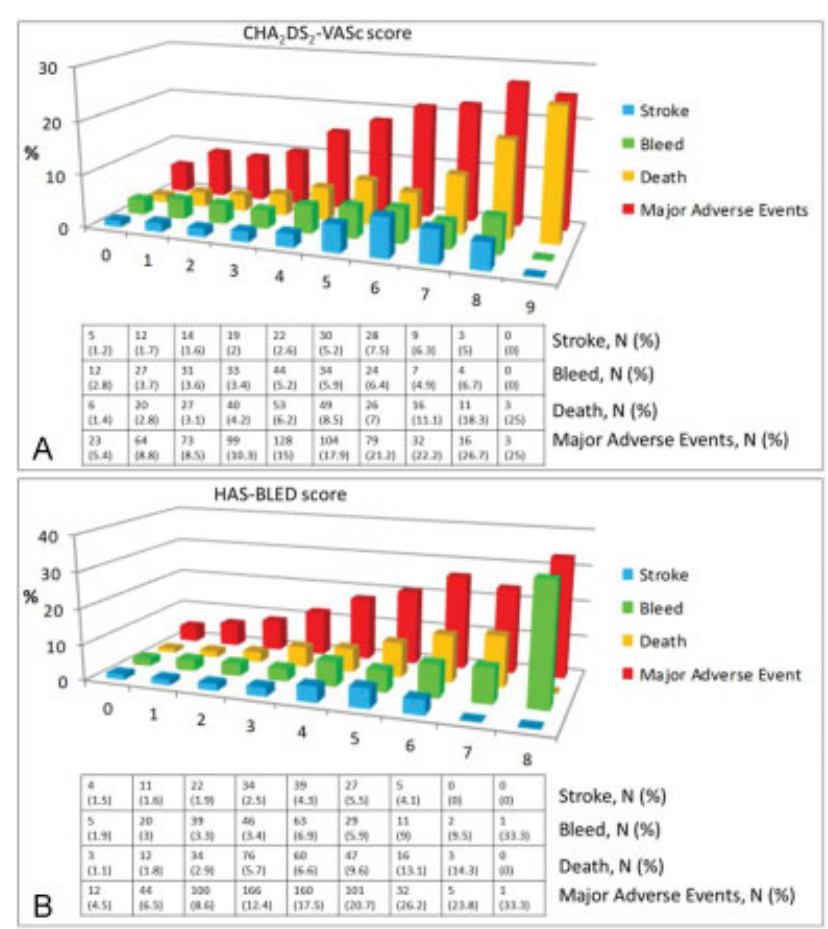

Fig. 1 Frequency (\%) of adverse events in patients with atrial fibrillation (AF) by $\mathrm{CHA}_{2} \mathrm{DS}_{2}$-VASc score (A). Frequency (\%) of adverse events in patients with atrial fibrillation (AF) by HAS-BLED score (B).

1.32) predicted higher mortality between discharge and day 90. $\mathrm{CHA}_{2} \mathrm{DS}_{2}$-VASc (adjusted OR, 1.08; 95\% CI, 1.01-1.16) and HAS-BLED scores (adjusted OR, 1.23; 95\% CI, 1.13-1.34) also predicted major adverse events by day 90 . Neither HAS-BLED score nor prescription of antiplatelet therapy was significantly associated with ISTH major bleeding.

\section{Evidence-Based Prevention of Stroke}

During hospitalization, anticoagulation was prescribed to $57.2 \%$ of the patient cohort and to $56.0 \%$ with a $\mathrm{CHA}_{2} \mathrm{DS}_{2}-$ VASc score of at least 1. During hospitalization, warfarin was the most commonly prescribed agent for stroke prevention in $\mathrm{AF}$ (55.2\%), followed by LMWH (15.7\%), unfractionated heparin (13.2\%), NOACs (5.8\%), and other thromboprophylaxis, including aspirin (11.1\%). Anticoagulation was prescribed to $62.1 \%$ upon discharge. At discharge, warfarin was the most commonly prescribed agent for stroke prevention in $\mathrm{AF}$ (58.7\%) followed by LMWH (10.7\%), NOACs (6.7\%), and other thromboprophylaxis including aspirin (23.9\%). Aspirin was prescribed to $17.1 \%$ of patients during hospitalization and $12.6 \%$ of patients at discharge.

The frequency of anticoagulation prescription remained relatively constant during the inpatient stay and at discharge across $\mathrm{CHA}_{2} \mathrm{DS}_{2}$-VASc scores ( - Fig. 2A). Use of anticoagulation decreased with an increasing HAS-BLED score (-Fig. 2B).

\section{Discussion}

We observed high all-cause mortality at 90 days (5.4\%) among hospitalized patients with AF. Cardiovascular causes of death were noted in $39.2 \%$ of inpatient deaths and in $12.2 \%$ of deaths taking place between discharge and day 90 . Anticoagulation prescription at discharge was associated with a $60 \%$ reduction in death between discharge and day 90 , after adjustment for confounding factors.

Table 3 Assessment of stroke and bleeding risk in patients receiving anticoagulation compared with those who were not

\begin{tabular}{|c|c|c|c|}
\hline Characteristic & $\begin{array}{l}\text { Anticoagulation } \\
N=3,105\end{array}$ & $\begin{array}{l}\text { No anticoagulation } \\
N=1,895\end{array}$ & $p$-Value \\
\hline Heart failure, $n(\%)$ & $670(21.6)$ & $287(15.2)$ & $<0.001$ \\
\hline Hypertension, $n$ (\%) & $2,190(70.5)$ & $1,338(70.6)$ & 0.95 \\
\hline Age $\geq 75$ y, $n(\%)$ & $1,076(34.7)$ & $721(38.1)$ & 0.02 \\
\hline Age 65-74 y, n (\%) & $905(29.2)$ & $534(28.2)$ & 0.46 \\
\hline Female, $n(\%)$ & $1,163(37.5)$ & $1,629(88.1)$ & 0.62 \\
\hline Diabetes, $n(\%)$ & $663(21.4)$ & $385(20.3)$ & 0.38 \\
\hline $\begin{array}{l}\text { Prior cerebrovascular accident or systemic } \\
\text { embolism, } n(\%)\end{array}$ & $551(17.8)$ & $330(17.5)$ & 0.93 \\
\hline Vascular disease, $n(\%)$ & $995(32.1)$ & $646(34.1)$ & 0.14 \\
\hline Renal dysfunction, $n(\%)$ & $403(13.0)$ & $280(14.8)$ & 0.49 \\
\hline Liver disease, $n$ (\%) & $108(3.5)$ & $119(6.3)$ & $<0.001$ \\
\hline Prior major bleed or predisposition to bleeding, $n(\%)$ & $770(24.8)$ & $742(39.2)$ & $<0.001$ \\
\hline Labile international normalized ratio, $n(\%)$ & $1,037(33.4)$ & $276(14.6)$ & $<0.001$ \\
\hline $\begin{array}{l}\text { Concomitant antiplatelet or nonsteroidal } \\
\text { anti-inflammatory drugs, } n(\%)\end{array}$ & $1,429(46.0)$ & $1,152(60.8)$ & $<0.001$ \\
\hline Alcohol intake $\geq 8$ servings per week, $n(\%)$ & $258(8.3)$ & $183(9.7)$ & 0.1 \\
\hline $\begin{array}{l}\text { Median } \mathrm{CHA}_{2} \mathrm{DS}_{2} \text {-VASc score } \\
\text { (interquartile range), points }\end{array}$ & $3(2-4)$ & $3(2-4)$ & 0.92 \\
\hline Median HAS-BLED score (interquartile range), points & $3(2-4)$ & $3(2-4)$ & $<0.001$ \\
\hline
\end{tabular}




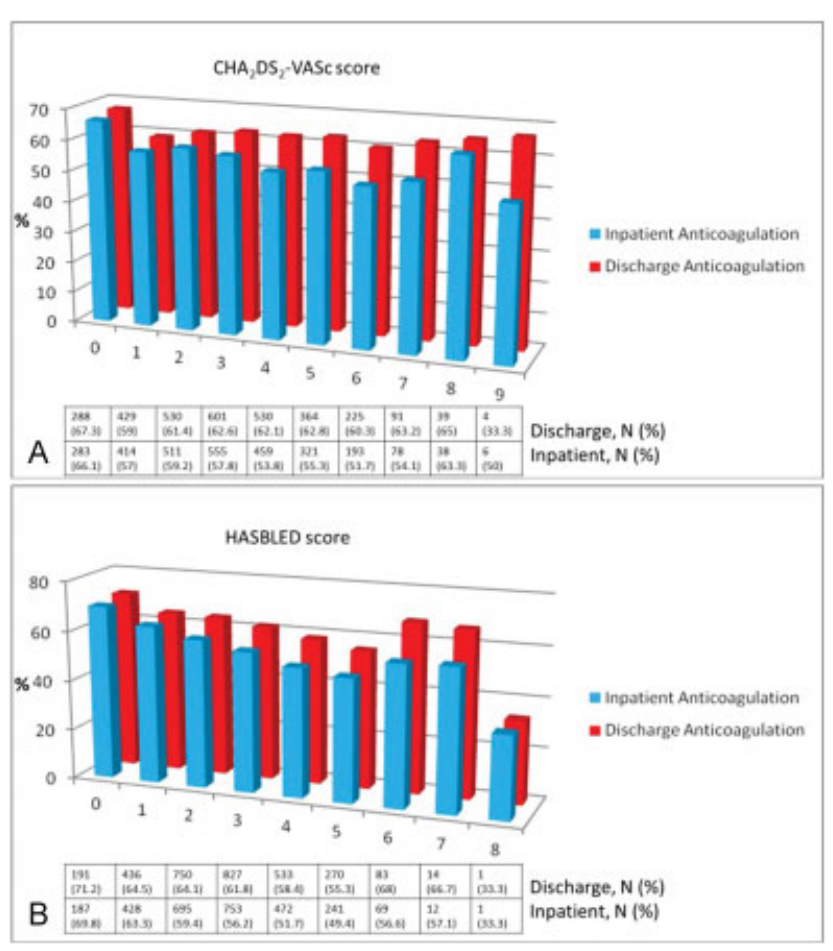

Fig. 2 Frequency (\%) of anticoagulation prescription during the inpatient stay and at discharge in patients with atrial fibrillation (AF) by $\mathrm{CHA}_{2} \mathrm{DS}_{2}$-VASc score (A). Frequency (\%) of anticoagulation prescription during the inpatient stay and at discharge in patients with atrial fibrillation (AF) by HAS-BLED score (B).

Epidemiological cohort studies ${ }^{2,12}$ and a systematic analysis of randomized controlled trial data ${ }^{4}$ estimate an annual adjusted mortality of 4 to $5 \%$ in patients hospitalized with AF. In the Medicare population of patients with $\mathrm{AF}$, annual mortality exceeds $16 \%{ }^{3}$ We observed a similarly high mortality in our tertiary care population of patients hospitalized with AF. In our observational study, we also observed that while cardiovascular disease was the most common cause of death, fatal stroke was relatively infrequent. Similarly, in the randomized (rivaroxaban vs. warfarin) ROCKET-AF trial, cardiovascular deaths occurred more than twice as often as strokes. Predictors of higher all-cause mortality included heart failure (hazard ratio, 1.51; 95\% CI, 1.33-1.70) and age greater than 75 years (hazard ratio, 1.69; 95\% CI, 1.51$1.90) .{ }^{13}$ Thus, further advances in anticoagulation strategies may have little effect on improving overall mortality in $\mathrm{AF}^{14}$ However, a cardiovascular risk factor management clinic for AF patients has been demonstrated to be clinically effective and cost-saving. ${ }^{15}$

Prescription of anticoagulation in AF patients at discharge was associated with a $60 \%$ reduction in all-cause mortality between discharge and day 90 , even after adjustment for confounding factors. This may have been due, in part, to selection of relatively healthy AF patients. Alternatively, anticoagulation may reduce both cardiovascular and noncardiovascular mortality in patients with $\mathrm{AF}$ via effects on other disease processes such as venous thromboembolism.
Our current inpatient study comprised patients with a higher medical acuity compared with our previous outpatient study, ${ }^{16}$ with respect to 90 -day all-cause mortality (5.4 vs. $1.2 \%$ ), stroke (2.8 vs. $1.6 \%$ ), and bleeding events (4.3 vs. $3.7 \%$ ). The inpatient population in the current study had a higher median HAS-BLED score (3 vs. 2) than our outpatient study. However, the frequency of anticoagulation prescription was higher for hospitalized AF patients at the time of discharge (62.1 vs. $46.9 \%$ ) compared with the rate in our previously published AF outpatient study. The findings of our study of current hospitalized patients with AF with respect to anticoagulation are consistent with those of the study of the ORBIT-AF Registry on 9,553 outpatients with AF. ${ }^{17}$

Anticoagulation prescription rates were low among patients with AF at our tertiary care center. In the international GARFIELD registry of AF patients, prescription rates for anticoagulation in those at high risk for stroke increased to $71 \%$ among the final $20 \%$ of participants who were enrolled in 2015 and 2016 (presented at the European Society of Cardiology Congress 2017). The findings in our current inpatient study and those in our prior outpatient study ${ }^{16}$ are consistent with the low rate of anticoagulation observed during the initial period of enrollment of GARFIELD AF in 2010 (composed entirely of non-U.S. centers). ${ }^{18}$ We hope that publication of the current study will provide an educational stimulus to U.S. providers to improve stroke prevention efforts in AF, because we link anticoagulation to prognosis.

There were multiple limitations to this retrospective, observational, administrative dataset. Our electronic data collection did not provide a complete profile of why anticoagulation was omitted in some of the hospitalized patients with AF. The database did not record the reason for hospitalization, which could have influenced prescription of anticoagulant therapy and the observed clinical outcomes. We could not distinguish whether anticoagulation was prescribed specifically for stroke prevention in AF or for some other indication. Our study database did not record data on International Normalized Ratio (INR) values or time within therapeutic range (TTR) for patients prescribed vitamin $\mathrm{K}$ antagonists. Despite adjusting for several variables, we may have missed confounding factors that could have impacted clinical outcomes. Cause of death was recorded as cardiovascular and noncardiovascular, and the database did not capture specific cardiovascular causes of death, such as sudden cardiac death. Finally, our study took place at a tertiary care center, and the results might not be representative of the patient populations at other institutions.

Our study provides a "real-world" analysis of the clinical characteristics, stroke and bleeding risks, anticoagulation practices, and clinical outcomes in 5,000 consecutive hospitalized patients with AF. Our analysis is strengthened by having complete (100\%) 90-day follow-up for the study cohort.

Recently, there has been increased emphasis on AF as a manifestation of systemic cardiovascular disease. Our study supports the impact of AF on cardiovascular mortality and highlights the magnitude of mortality reduction when $\mathrm{AF}$ patients are discharged on anticoagulation. 


\section{Conclusion}

Hospitalized patients with AF have high all-cause mortality at 90 days. Anticoagulation prescription at discharge was associated with a $60 \%$ reduction in death between discharge and day 90 . Hospitalization represents a special opportunity to implement cardiovascular risk reduction strategies, especially anticoagulation.

\section{Conflicts of Interest}

Dr. Piazza has received research grant support from Bristol Myers Squibb, Daiichi Sankyo, the Thrombosis Research Institute, and Janssen and consulting fees from Merck. Dr. Goldhaber has received research grant support from Boehringer Ingelheim, Bristol-Myers Squibb, Daiichi Sankyo, the Thrombosis Research Institute, and Janssen and consulting fees from Boehringer Ingelheim, BristolMyers Squibb, Daiichi Sankyo, Eisai, Merck, Portola, and sanofi-aventis. Drs. Hurwitz and Hohlfelder and Ms. Jenkins, Ms. Harrigan, and Mr. Fanikos have no conflicts of interest to disclose.

\section{Funding}

This study was funded in part by a research grant from the Bristol-Myers Squibb-Pfizer Alliance. The sponsor of the study had no role in study design, data collection, data analysis, data interpretation, or writing of the report.

\section{Authors' Contributions}

Drs. Piazza and Hurwitz had full access to all the data in the study and take full responsibility for the integrity of the data and the accuracy of the data analysis. Drs. Piazza (BWH, Cardiovascular Division) and Hurwitz (BWH, Center for Clinical Investigation) conducted and are responsible for the data analysis.

\section{References}

1 Benjamin EJ, Wolf PA, D'Agostino RB, Silbershatz H, Kannel WB, Levy D. Impact of atrial fibrillation on the risk of death: the Framingham Heart Study. Circulation 1998;98(10):946-952

2 Fauchier L, Villejoubert O, Clementy N, et al. Causes of death and influencing factors in patients with atrial fibrillation. Am J Med 2016;129(12):1278-1287

3 Freeman JV, Wang Y, Akar J, Desai N, Krumholz H. National trends in atrial fibrillation hospitalization, readmission, and mortality for Medicare beneficiaries, 1999-2013. Circulation 2017;135(13): $1227-1239$

4 Gómez-Outes A, Lagunar-Ruíz J, Terleira-Fernández AI, CalvoRojas G, Suárez-Gea ML, Vargas-Castrillón E. Causes of death in anticoagulated patients with atrial fibrillation. J Am Coll Cardiol 2016;68(23):2508-2521
5 Odutayo A, Wong CX, Hsiao AJ, Hopewell S, Altman DG, Emdin CA. Atrial fibrillation and risks of cardiovascular disease, renal disease, and death: systematic review and meta-analysis. BMJ 2016; 354:i4482

6 Hart RG, Pearce LA, Aguilar MI. Meta-analysis: antithrombotic therapy to prevent stroke in patients who have nonvalvular atrial fibrillation. Ann Intern Med 2007;146(12):857-867

7 Lip G, Freedman B, De Caterina R, Potpara TS. Stroke prevention in atrial fibrillation: Past, present and future. Comparing the guidelines and practical decision-making. Thromb Haemost 2017;117 (07):1230-1239

8 Harris PA, Taylor R, Thielke R, Payne J, Gonzalez N, Conde JG. Research electronic data capture (REDCap)-a metadata-driven methodology and workflow process for providing translational research informatics support. J Biomed Inform 2009;42(02): 377-381

9 Lip GY, Halperin JL. Improving stroke risk stratification in atrial fibrillation. Am J Med 2010;123(06):484-488

10 Pisters R, Lane DA, Nieuwlaat R, de Vos CB, Crijns HJ, Lip GY. A novel user-friendly score (HAS-BLED) to assess 1-year risk of major bleeding in patients with atrial fibrillation: the Euro Heart Survey. Chest 2010;138(05):1093-1100

11 Schulman S, Kearon C; Subcommittee on Control of Anticoagulation of the Scientific and Standardization Committee of the International Society on Thrombosis and Haemostasis. Definition of major bleeding in clinical investigations of antihemostatic medicinal products in non-surgical patients. J Thromb Haemost 2005;3(04):692-694

12 Bassand JP, Accetta G, Camm AJ, et al; GARFIELD-AF Investigators. Two-year outcomes of patients with newly diagnosed atrial fibrillation: results from GARFIELD-AF. Eur Heart J 2016;37(38): 2882-2889

13 Pokorney SD, Piccini JP, Stevens SR, et al; ROCKET AF Steering Committee \& Investigators; ROCKET AF Steering Committee Investigators. Cause of death and predictors of all-cause mortality in anticoagulated patients with nonvalvular atrial fibrillation: data from ROCKET AF. J Am Heart Assoc 2016;5(03): e002197

14 Staerk L, Sherer JA, Ko D, Benjamin EJ, Helm RH. Atrial fibrillation: epidemiology, pathophysiology, and clinical outcomes. Circ Res 2017;120(09):1501-1517

15 Pathak RK, Evans M, Middeldorp ME, et al. Cost-effectiveness and clinical effectiveness of the risk factor management clinic in atrial fibrillation. JACC Clin Electrophysiol 2017;3(05):436-447

16 Piazza G, Karipineni N, Goldberg HS, Jenkins KL, Goldhaber SZ. Underutilization of anticoagulation for stroke prevention in atrial fibrillation. J Am Coll Cardiol 2016;67(20):2444-2446

17 Hess PL, Kim S, Fonarow GC, et al; Outcomes Registry for Better Informed Treatment of Atrial Fibrillation (ORBIT-AF) Patients and Investigators. Absence of oral anticoagulation and subsequent outcomes among outpatients with atrial fibrillation. Am J Med 2017;130(04):449-456

18 Kakkar AK, Mueller I, Bassand JP, et al; GARFIELD Registry Investigators. Risk profiles and antithrombotic treatment of patients newly diagnosed with atrial fibrillation at risk of stroke: perspectives from the international, observational, prospective GARFIELD registry. PLoS One 2013;8(05):e63479 